6-1-1992

\title{
Reliability Analysis of Structural Ceramic Components Using a Three-parameter Weibull Distribution
}

\author{
Stephen F. Duffy \\ Cleveland State University, s.duffy@csuohio.edu \\ Lynn M. Powers \\ Alois Starlinger \\ Lewis Research Center, Cleveland, $\mathrm{OH}$
}

Follow this and additional works at: https://engagedscholarship.csuohio.edu/encee_facpub

Part of the Aerospace Engineering Commons

How does access to this work benefit you? Let us know!

\section{Recommended Citation}

Duffy, Stephen F.; Powers, Lynn M.; and Starlinger, Alois, "Reliability Analysis of Structural Ceramic Components Using a Three-parameter Weibull Distribution" (1992). Civil and Environmental Engineering Faculty Publications. 3.

https://engagedscholarship.csuohio.edu/encee_facpub/3

This Report is brought to you for free and open access by the Civil and Environmental Engineering at EngagedScholarship@CSU. It has been accepted for inclusion in Civil and Environmental Engineering Faculty Publications by an authorized administrator of EngagedScholarship@CSU. For more information, please contact library.es@csuohio.edu. 


\section{$1-2982$ \\ $E-6749$}

NASA Technical Memorandum 105370

\section{Reliability Analysis of Structural Ceramic Components Using a Three-Parameter Weibull Distribution}

Stephen F. Duffy and Lynn M. Powers

Cleveland State University

Cleveland, Ohio

and

Alois Starlinger

Lewis Research Center

Cleveland, Ohio

Prepared for the

37th International Gas Turbine and Aeroengine Congress and Exposition sponsored by the American Society of Mechanical Engineers

Cologne, Germany, June 1-4, 1992 


\title{
RELIABILITY ANALYSIS OF STRUCTURAL CERAMIC COMPONENTS USING A
}

\section{THREE-PARAMETER WEIBULL DISTRIBUTION}

\author{
Stephen F. Duffy ${ }^{*}$ and Lynn M. Powers ${ }^{*}$ \\ Cleveland State University \\ Cleveland, Ohio 44115 \\ and \\ Alois Starlinger ${ }^{\dagger}$ \\ National Aeronautics and Space Administration \\ Lewis Research Center \\ Cleveland, Ohio 44135
}

\begin{abstract}
This paper describes nonlinear regression estimators for the three-parameter Weibull distribution. Issues relating to the bias and invariance associated with these estimators are examined numerically using Monte Carlo simulation methods. The estimators were used to extract parameters from sintered silicon nitride failure data. A reliability analysis was performed on a turbopump blade utilizing the three-parameter Weibull distribution and the estimates from the sintered silicon nitride data.
\end{abstract}

\section{INTRODUCTION}

To date, most reliability analyses performed on structural components fabricated from ceramic materials have utilized the twoparameter form of the Weibull distribution. The use of a twoparameter Weibull distribution to characterize the random nature of material strength implies a nonzero probability of failure for the full range of applied stress. This represents a conservative design assumption when analyzing structural components. A three parameter form of the Weibull distribution is available. The additional parameter is a threshold stress that allows for zero probability of failure when applied stress is at or below the threshold value. By employing the concept of a threshold stress, design engineers can effectively tailor the design of a component to optimize structural reliability.

Difficulties in estimating parameters as well as a lack of strength data with corresponding fractographic analysis has limited the use of this distribution. Several authors (including Weibull, 1939; Weil and Daniel, 1964; and Schneider and Palazotto, 1979) have proposed estimation methods for the three-parameter distribution. For various reasons these techniques have not been widely utilized. The nonlinear regression method proposed by Margetson and Cooper (1984) is adopted here to establish estimators for the three-parameter Weibull formulation. Estimators are applied using failure data obtained from the open literature. Specifically, Weibull parameters are estimated from failure data

*NASA Resident Research Associate at Lewis Research Center.

$\dagger_{\text {National Research Council - NASA Research Associate at }}$

Lewis Research Center. reported by Chao and Shetty (1991). The data were generated from test specimens fabricated from a monolithic silicon nitride. Strength tests were conducted on this material using three-point bend, fourpoint bend, and pressurized-disk, specimen geometries. Here the Weibull parameters are estimated from the four-point bend test data, and failure data from the three-point bend tests and pressurized-disk tests are subsequently used to challenge structural reliability predictions made for these latter two geometries. To conduct structural reliability analyses, the three-parameter Weibull distribution was embedded in a reliability model known as the principle of independent action (PIA). We point out that the threeparameter form of the Weibull distribution can be extended to Batdorf's $(1974,1978)$ model and reliability models proposed for ceramic matrix composites (see Duffy et al., 1991; or Thomas and Wetherhold, 1991). All reliability computations presented here were made utilizing the integrated design program CARES (Ceramic Analysis and Reliability Evaluation of Structures) (Nemeth et al., 1990).

In general, the objective of parameter estimation is the derivation of functions (or estimators) that are dependent on the failure data and that yield, in some sense, optimum estimates of the underlying population parameters. Various performance criteria can be applied to ensure that optimized estimates are obtained consistently. Two important performance criteria are estimate invariance and estimate bias. An estimator is invariant if the bias associated with the estimated value is independent of the true parameters that characterize the underlying population. Bias is a measure of deviation of the estimated parameter from the true population parameter. Here the functional value of an estimator is a point estimate (in contrast to an interval estimate) of the true population parameter. The values of the point estimates computed from a number of samples obtained from a population will vary from sample to sample. A sample is defined as a collection (i.e., more than one) of observations taken from a specified population, and a population represents the totality of all possible observations about which statistical inferences could be made. In this paper, the observations are the failure strengths of test specimens fabricated from ceramic materials. The issues of bias and invariance and their relationship to the functions proposed by Margetson and Cooper (1984) are explored numerically. In the numerical studies, distributions of the point estimates are obtained by taking numerous samples from the population and computing point estimates as a function of sample size. 
If the mean of a distribution of such estimates is equal to the value of the true parameter for a given sample size, the associated estimator is said to be unbiased. If an estimator yields biased results, the value of the individual estimates can be corrected if the estimators are invariant (see Thoman et al., 1969, for the procedure associated with two-parameter maximum-likelihood estimators).

The Monte Carlo simulations that are presented later demonstrate that the functions are neither invariant nor unbiased.

\section{ESTIMATING WEIBULL DISTRIBUTION PARAMETERS}

Weibull $(1939,1951)$ proposed the first probabilistic model that accounted for scatter in failure strength and the size effect encountered in structural components fabricated from brittle materials. His approach is based on the weakest link theory (WLT) attributed to Midgley and Pierce (1926). This earlier research (sponsored by the textile industry) focused on modeling yarn strength. Unlike Midgley and Pierce, who assumed a Gaussian distribution for yarn strength, Weibull proposed a unique probability density function for failure strength that now bears his name. Weibull's three-parameter probability density function has the following form

$$
f(x)=\left(\frac{\alpha}{\eta}\right)\left(\frac{x-\lambda}{\eta}\right)^{(\alpha-1)} \exp \left(-\left(\frac{x-\lambda}{\eta}\right)^{\alpha}\right)
$$

for a continuous random variable $\mathrm{x}$, when $0 \leq \lambda<\mathrm{x}$, and

$$
f(x)=0
$$

for $x \leq \lambda$. The cumulative distribution function is given by the expression

$$
F(x)=1-\exp \left(-\left(\frac{x-\lambda}{\eta}\right)^{\alpha}\right)
$$

for $x>\lambda$, and

$$
\mathrm{F}(\mathrm{x})=0
$$

for $x \leq \lambda$. Here $\alpha(>0)$ is the Weibull modulus (or the shape parameter), $\eta(>0)$ is the scale parameter, and $\lambda(>0)$ is the threshold parameter. When applied to analyses of structural components, the random variable $\mathrm{x}$ usually represents a component of the Cauchy stress tensor or an invariant of this tensor. For a uniaxial stress field in a homogeneous isotropic material, application of Weibull's theory yields the following expression for the probability of failure

$$
\begin{aligned}
\boldsymbol{P} & =1-\exp \left(-\int_{V}\left(\frac{\sigma-\gamma}{\beta}\right)^{\alpha} \mathrm{dV}\right) \\
& =1-\exp (-\mathrm{B}) \quad \sigma>\gamma
\end{aligned}
$$

where

$$
B=\int_{V}\left(\frac{\sigma-\gamma}{\beta}\right)^{\alpha} d V
$$

and

$$
\mathcal{P}=0 \quad \sigma \leq \gamma
$$

Note that $\alpha, \beta$, and $\gamma$ are material parameters and will not depend on the geometry of the test specimen. In this context $\beta$ has the dimension of (stress) $\cdot(\text { volume })^{1 / \alpha}, \gamma$ has the dimension of stress, and $\alpha$ is dimensionless.

Certain monolithic ceramics have exhibited threshold behavior (e.g., Quinn, 1989; Chao and Shetty, 1991). It is anticipated that ceramic matrix composites will similarly exhibit this behavior (Duffy et al., 1991). Threshold behavior is demonstrated if the failure data displays a nonlinear behavior when the ranked probability of failure $\left(\mathcal{P}_{i}\right)$ is represented as a function of the corresponding failure values. Careful fractography must yield clear evidence that only one type of defect is causing failure. Thus, the fractographic analysis must demonstrate that the nonlinear behavior of the failure data is not the result of competing failure mechanisms. When experimental data indicates the existence of a threshold stress, a threeparameter Weibull distribution should be employed in the stochastic failure analysis of structural components. However, the threeparameter form of the Weibull distribution has been somewhat ignored as a result of difficulties encountered in extracting estimates of the parameters from experimental data. Margetson and Cooper (1984) proposed a relatively simple nonlinear regression method to estimate the three distribution parameters. Regression analysis postulates a relationship between two variables. In an experiment, typically one variable can be controlled (the independent variable) while the response variable (or dependent variable) is uncontrolled. In simple failure experiments the material dictates the strength at failure, indicating that the failure stress is the response variable. The ranked probability of failure $\left(\boldsymbol{P}_{\mathrm{i}}\right)$ can be controlled by the experimentalist since it is functionally dependent on the sample size (N). After numbering the observed failure stresses $\left(\sigma_{1}, \sigma_{2}, \sigma_{3}, \ldots\right.$, $\left.\sigma_{\mathrm{N}}\right)$ in ascending order, and specifying

$$
\boldsymbol{P}_{\mathrm{i}}\left(\sigma_{\mathrm{i}}\right)=(\mathrm{i}-0.5) / \mathrm{N}
$$

then clearly the ranked probability of failure for a given stress leve can be influenced by increasing or decreasing the sample size. The procedure proposed by Margetson and Cooper (1984) adopts this philosophy. They assume that the specimen failure stress is the dependent variable, and the associated ranked probability of failure becomes the independent variable.

Using Eq. (5), an expression can be obtained relating the ranked probability of failure $\left(\mathcal{P}_{i}\right)$ to an estimate of the failure strength $\left(\hat{\sigma}_{\mathrm{i}}\right)$. Assuming uniaxial stress conditions in a test specimen with a unit volume, Eq. (5) yields

$$
\hat{\sigma}_{\mathrm{i}}=\hat{\gamma}+\hat{\beta}\left[\ln \left(1 / 1-\boldsymbol{P}_{\mathrm{i}}\right)\right]^{1 / \hat{\alpha}}
$$

where $\hat{\sigma}_{\mathrm{i}}$ is an estimate of the ranked failure stress. In addition,

$\hat{\alpha}, \hat{\beta}$, and $\hat{\gamma}$ are estimates of the shape parameter $(\alpha)$, the scale parameter $(\beta)$, and the threshold parameter $(\gamma)$, respectively. Defining the residual as 


$$
\delta_{\mathrm{i}}=\hat{\sigma}_{\mathrm{i}}-\sigma_{\mathrm{i}}
$$

where $\sigma_{i}$ is the i'th ranked failure stress obtained from actual test data, then the sum of the squared residuals is expressed as

$$
\sum_{i=1}^{N}\left(\delta_{i}\right)^{2}=\sum_{i=1}^{N}\left(\hat{\gamma}+\hat{\beta} \mathrm{W}_{\mathrm{i}}^{1 / \hat{\alpha}}-\sigma_{\mathrm{i}}\right)^{2}
$$

Here the notation of Margetson and Cooper (1984) is adopted where

$$
\mathrm{W}_{\mathrm{i}}=\ln \left(1 / 1-\boldsymbol{P}_{\mathrm{i}}\right)
$$

Note that the forms of $\hat{\sigma}_{i}$ and $W_{i}$ change with specimen geometry (see the discussion in a later section relating to the fourpoint bend specimen geometry). It should be apparent that the objective of this method is to obtain parameter estimates that minimize the sum of the squared residuals. Setting the partial derivatives of the sum of the squares of the residuals with respect to $\hat{\alpha}, \hat{\beta}$, and $\hat{\gamma}$ equal to zero yields the following three expressions

$$
\begin{gathered}
\hat{\beta}=\frac{N\left(\sum_{i=1}^{N} \sigma_{i}\left(W_{i}\right)^{1 / \hat{\alpha}}\right)-\left(\sum_{i=1}^{N} \sigma_{i}\right)\left(\sum_{i=1}^{N}\left(W_{i}\right)^{1 / \hat{\alpha}}\right)}{N \sum_{i=1}^{N}\left(W_{i}\right)^{2 / \hat{\alpha}}-\left(\sum_{i=1}^{N}\left(W_{i}\right)^{1 / \hat{\alpha}}\right)\left(\sum_{i=1}^{N}\left(W_{i}\right)^{1 / \hat{\alpha}}\right)} \\
\hat{\gamma}=\frac{\left(\sum_{i=1}^{N}\left(W_{i}\right)^{2 / \hat{\alpha}}\right)\left(\sum_{i=1}^{N} \sigma_{i}\right)-\left(\sum_{i=1}^{N} \sigma_{i}\left(W_{i}\right)^{1 / \hat{\alpha}}\right)\left(\sum_{i=1}^{N}\left(W_{i}\right)^{1 / \hat{\alpha}}\right)}{N \sum_{i=1}^{N}\left(W_{i}\right)^{2 / \hat{\alpha}}-\left(\sum_{i=1}^{N}\left(W_{i}\right)^{1 / \hat{\alpha}}\right)\left(\sum_{i=1}^{N}\left(W_{i}\right)^{1 / \hat{\alpha}}\right)}
\end{gathered}
$$

and

$$
\begin{gathered}
\mid \sum_{\mathrm{i}=1}^{\mathrm{N}} \sigma_{\mathrm{i}}\left(\mathrm{W}_{\mathrm{i}}\right)^{1 / \hat{\alpha}} \ln \left(\mathrm{W}_{\mathrm{i}}\right)-\hat{\gamma} \sum_{\mathrm{i}=1}^{\mathrm{N}}\left(\mathrm{W}_{\mathrm{i}}\right)^{1 / \hat{\alpha}} \ln \left(\mathrm{W}_{\mathrm{i}}\right) \\
-\hat{\beta} \sum_{\mathrm{i}=1}^{\mathrm{N}}\left(\mathrm{W}_{\mathrm{i}}\right)^{2 / \hat{\alpha}} \ln \left(\mathrm{W}_{\mathrm{i}}\right) \mid \leq \kappa_{\mathrm{conv}}
\end{gathered}
$$

in terms of the parameter estimates. The solution of this system of equations is iterative. The third expression is used to check convergence of the iterative solution. The initial solution vector for this system is determined after assuming $\hat{\alpha}=1$. Then $\hat{\beta}$ is computed from Eq. (13) and $\hat{\gamma}$ is calculated from Eq. (14). The values of these parameter estimates are then inserted into Eq. (15) to determine if the convergence criterion is satisfied to within some predetermined tolerance $\left(\kappa_{\text {conv }}\right)$. If this expression is not satisfied, $\hat{\alpha}$ is updated and a new iteration is conducted. This procedure continues until a set of parameter estimates are determined that satisfy Eq. (15).

\section{BIAS AND INVARIANCE}

Issues relating to estimate bias and invariance are examined numerically using Monte Carlo simulation methods. In this study uniform random numbers are generated in groups of $\mathrm{N}$ (which characterizes the sample size), and this is repeated 10000 times for each value N. Each group of uniform random numbers is generated on the interval 0 to 1 using the Cray random number function RANGET. The uniform random number is converted to a strength observation by employing the inverse of the three-parameter Weibull distribution for failure strength given in Eq. (9). Defining $\left(\mathrm{S}_{\mathrm{i}}\right)_{\mathrm{N}}$ as the $\mathrm{i}$ 'th random number on the interval 0 to 1 in a sample of size $\mathrm{N}$, then the $\mathrm{i}$ 'th failure strength is

$$
\left(\sigma_{\mathrm{i}}\right)_{\mathrm{N}}=\gamma+\beta\left[\ln \left(\frac{1}{1-\left(\mathrm{S}_{\mathrm{i}}\right)_{\mathrm{N}}}\right)\right]^{1 / \alpha}
$$

where $\alpha, \beta$, and $\gamma$ are the true distribution parameters of an infinite population characterized by a three-parameter Weibull distribution. Again, uniaxial stress conditions are imposed on a specimen of unit volume. However, this method can be extended to other specimen geometries as well.

Once a sample of $\mathrm{N}$ random numbers is generated and converted to failure strength observations, the estimators described by Eq. (13) through (15) are used to obtain the point estimates

$\hat{\alpha}, \hat{\beta}$, and $\hat{\gamma}$. Percentile distributions of the point estimates, as well as a mean value of the point estimates, can be constructed by repeating this sampling procedure for each value of $\mathrm{N}$. Here the Monte Carlo simulations are carried out 10000 times for each $\mathrm{N}$. The arithmetic mean of each estimated parameter is a measure of the bias associated with the estimator in determining that parameter, and is usually characterized as a function of the sample size (N). This is depicted graphically in Fig. 1. In this figure the vertical axes represent a ratio of the point estimate value to the parameter true value used to generate the failure observations. The true population parameters are arbitrarily chosen, with $\alpha=1.75, \beta$ $=1000$, and $\lambda=300$. The horizontal axes represent the sample size N. Note that for all three estimators the mean value of the ratio approaches 1 for large values of $\mathrm{N}$. Thus, each estimator exhibits the attractive property of decreasing bias with increasing sample size. However, the arithmetic mean associated with each parameter is not invariant with respect to the underlying population parameter. This is evident in Fig. 2 which depicts the arithmetic mean values of the parameter estimates from the previous example along with arithmetic mean values from a second example. For the second sample, the true population parameter $\alpha$ has been increased such that $\alpha=2.75$, and the other values of the true parameters are unchanged. Clearly the arithmetic means associated with the Weibull modulus $(\alpha)$, the scale parameter $(\beta)$, and threshold stress $(\gamma)$ change for sample sizes of less than 100 . If the mean values remained invariant, then the three curves in each graph in Fig. 2 would coincide regardless of the values assumed for the true population parameters. This lack of invariance precludes unbiasing the point estimates obtained using this method. If the estimators were invariant, the bias could be removed in a systematic fashion using the method outlined by Thoman et al. (1969) for the maximum-likelihood estimate of the Weibull modulus. The authors indicate that the ratio associated with the two-parameter maximumlikelihood estimator for the scale parameter is also not invariant with respect to the underlying population parameters. However, Thoman et al. (1969) were able to construct a function that contained the ratio associated with the scale parameter and the estimate of the Weibull modulus, but was not dependent on the true population parameters. This function enabled Thoman et al. (1969) to establish unbiasing factors and confidence bounds for the 


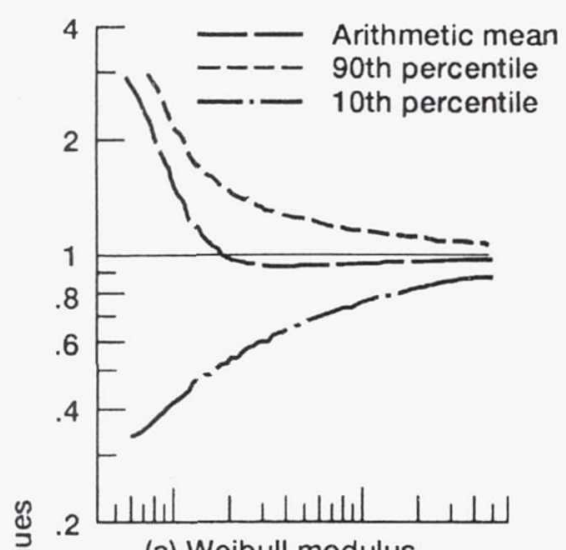

(a) Weibull modulus.

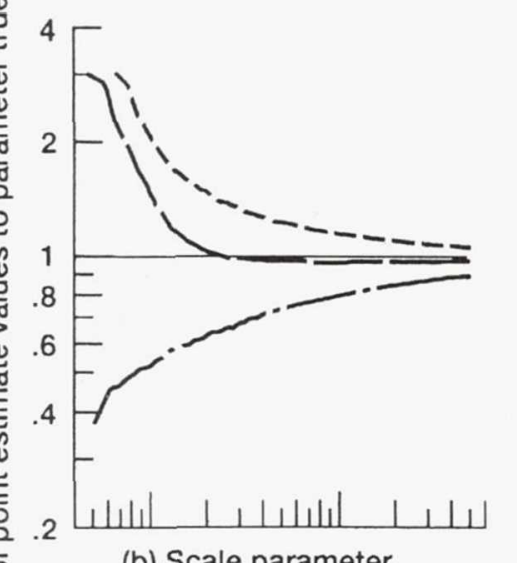

(b) Scale parameter.

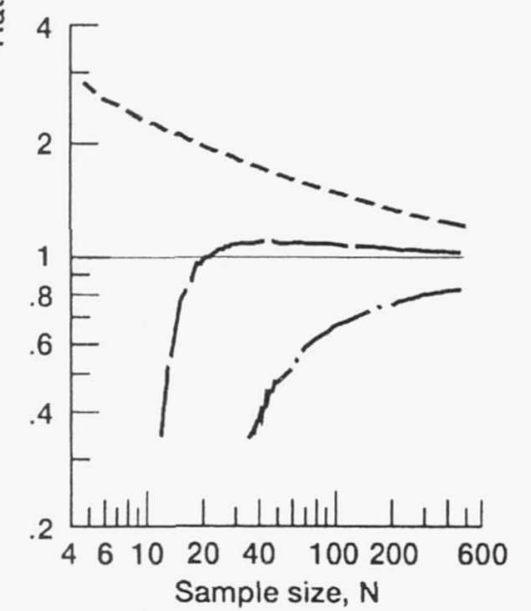

(c) Threshold stress.

Figure 1.-Arithmetic mean values and percentile distributions of the ratio of the point estimate to the true population parameter for each estimator $(\alpha=1.75$, $\beta=1000, \gamma=300$ ).

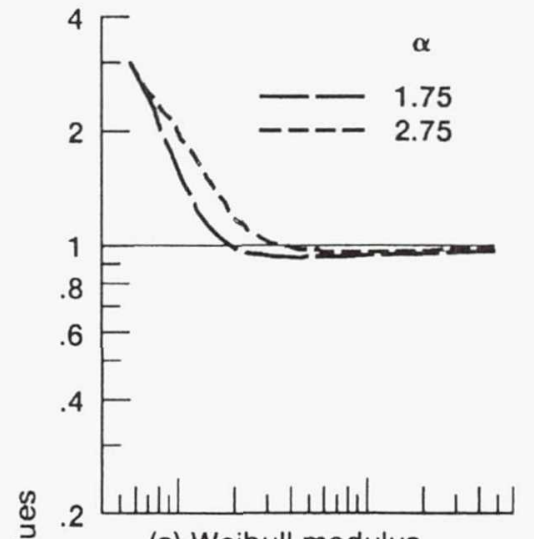

(a) Weibull modulus.

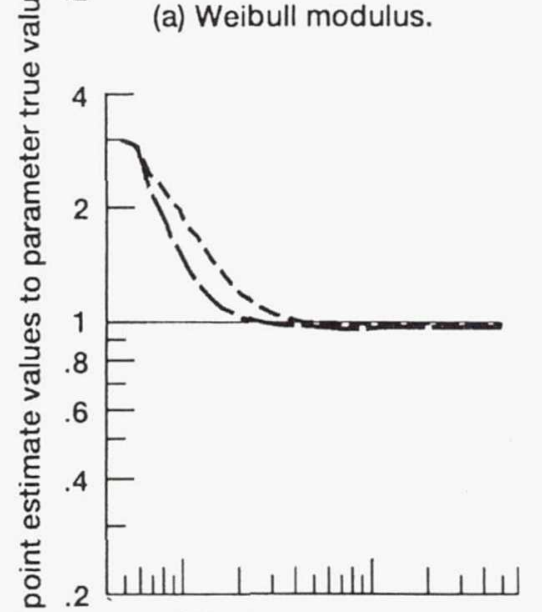

(b) Scale parameter.

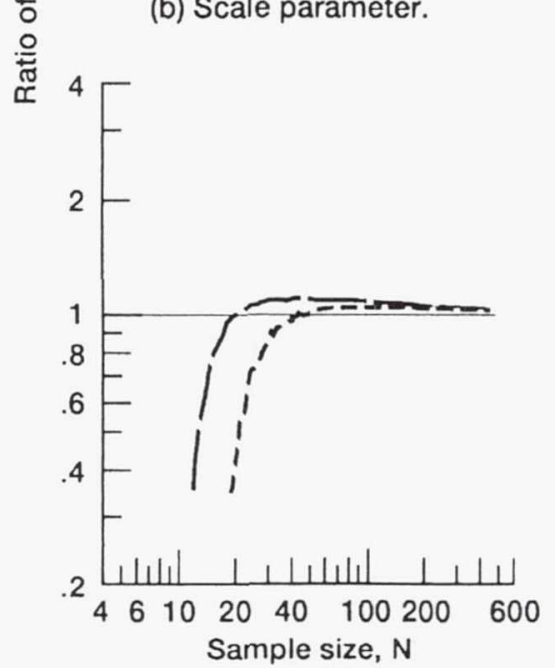

(c) Threshold stress.

Figure 2.-Arithmetic mean values of the ratio of the point estimate to the true population parameter for each estimator $(\beta=1000, \gamma=300)$.

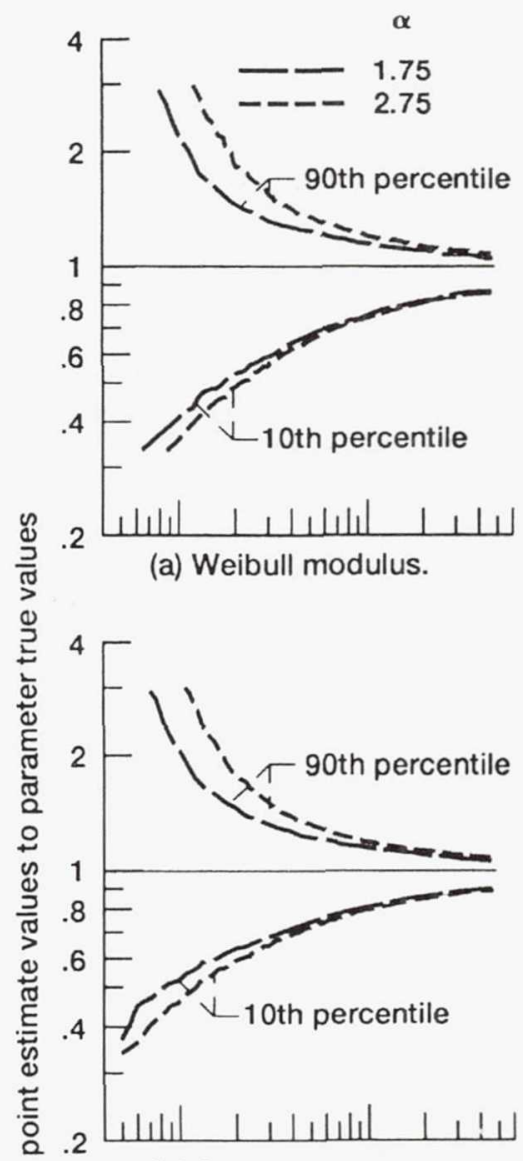

(b) Scale parameter.

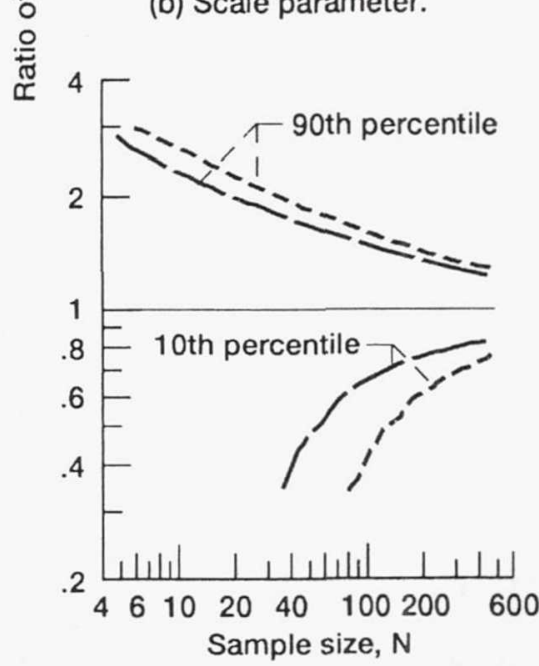

(c) Threshold stress.

Figure 3.-Percentile distributions of the ratio of the point estimate to the true population parameter for each estimator $(\beta=1000, \gamma=300)$. 
maximum-likelihood estimate of the two-parameter scale parameter. Similar functions for the nonlinear regression estimators discussed in this paper have not been developed. Thus, removing the bias associated with these estimators is not possible, and the design engineer should recognize that the amount of bias may be significant for small sample sizes.

Along with the mean value, the 10th and 90th percentile distributions are depicted for each estimator in Fig. 1. These percentile distributions are related to confidence bounds for a point estimate. The percentile distributions are obtained by ranking in order (from lowest to highest value) the ratios of point estimates to the true value of the distribution parameter. In this case the 10 th percentile distribution represents the ratio associated with the 1000 th ranked value. Hence, 999 ratios had lesser values. Similarly, the 90th percentile distribution represents the ratio associated with the 9000th ranked value. If the number of samples was increased from 10000 to infinity, then these ranked values would yield the exact confidence bounds for the estimators. Note that for these estimators the confidence bounds narrow with increasing sample size $(\mathrm{N})$. This is indicated by the decreasing separation in the percentile distributions. However, the percentile distributions are not invariant with respect to the true population parameters. Again, increasing $\alpha$ from 1.75 to 2.75 affected the percentile distributions (Fig. 3). This precludes the computation of confidence bounds on parameter estimates since the value of the true population parameter (the quantity being estimated) would have to be known a priori.

\section{APPLICATION - PARAMETER ESTIMATION AND RELIABILITY ANALYSIS}

In this section, parameters from the sintered silicon nitride (grade SNW-1000, GTE Wesgo Division) data presented in Chao

\section{TABLE I. - ESTLMATED PARAMETERS FOR SILICON NITRIDE}

\begin{tabular}{|c|c|c|c|}
\hline \multirow{2}{*}{$\begin{array}{l}\text { Specimen } \\
\text { number }\end{array}$} & \multicolumn{3}{|c|}{ Strength, $\mathrm{MPa}$} \\
\hline & Three-point bend & Four-point bend & Pressurized disk \\
\hline 1 & 715.6 & 613.9 & 549.7 \\
\hline 2 & 729.6 & 623.4 & 575.5 \\
\hline 3 & 741.0 & 639.3 & 587.4 \\
\hline 4 & 758.6 & 642.1 & 622.0 \\
\hline 5 & 771.4 & 6.53 .8 & 636.7 \\
\hline 6 & 773.1 & 662.4 & 639.3 \\
\hline 7 & 824.2 & 669.5 & 642.6 \\
\hline 8 & 830.4 & 672.8 & 646.3 \\
\hline 9 & 832.8 & 681.3 & 659.3 \\
\hline 10 & 863.2 & 682.0 & 659.6 \\
\hline 11 & 868.2 & 699.0 & 660.4 \\
\hline 12 & 870.9 & 714.5 & 661.4 \\
\hline 13 & 878.3 & 717.4 & 667.8 \\
\hline 14 & 881.1 & 725.5 & 668.9 \\
\hline 15 & 899.4 & 741.6 & 670.8 \\
\hline 16 & 900.6 & 744.9 & 684.8 \\
\hline 17 & 905.0 & 751.0 & 686.2 \\
\hline 18 & 913.8 & 761.7 & 691.3 \\
\hline 19 & 916.8 & 763.9 & 693.8 \\
\hline 20 & 928.0 & 774.2 & 698.1 \\
\hline 21 & 931.0 & 791.6 & 706.9 \\
\hline 22 & 934.6 & 795.2 & 7181 \\
\hline 23 & 935.1 & 829.8 & 718.8 \\
\hline 24 & 941.1 & 838.4 & 726.4 \\
\hline 25 & 941.6 & 856.4 & 732.3 \\
\hline 26 & 949.1 & 868.3 & 738.1 \\
\hline 27 & 951.6 & 882.9 & 748.2 \\
\hline 28 & 953.8 & $\cdots$ & 771.5 \\
\hline 29 & 956.5 & $\cdots+$. & 780.7 \\
\hline 30 & 979.9 & $\cdots+.$. & 786.3 \\
\hline 31 & $-\cdots$ & $\cdots$ & 796.2 \\
\hline 32 & ...... & ….. & 811.6 \\
\hline
\end{tabular}

and Shetty (1991) are estimated. The four-point bend, the threepoint bend, and the pressurized-disk data are listed in Table I. Focusing on the four-point bend specimen, the support span for this test fixture was $40.373 \mathrm{~mm}$ and the inner load span was $19.622 \mathrm{~mm}$. The cross sections of the test specimens were $4.0138 \mathrm{~mm}$ wide and $3.1106 \mathrm{~mm}$ in height. ${ }^{1}$ All failures occurred within the $19.6-\mathrm{mm}$ gage section. Thus, each specimen is assumed to be subjected to pure bending. Under this assumption, Eq. (6) becomes (see Weil and Daniel, 1964)

$$
\mathrm{B}=\left(\frac{\mathrm{V}}{2(\alpha+1)}\right)\left(\frac{\sigma-\gamma}{\sigma}\right)\left(\frac{\sigma-\gamma}{\beta}\right)^{\alpha}
$$

where

$$
\mathrm{V}=\mathrm{bh} \mathrm{l}=243.0 \mathrm{~mm}^{3}
$$

and $\sigma(=\mathrm{Mc} / \mathrm{I})$ is the outer fiber stress, assuming that the material behaves in a linear elastic fashion. Chao and Shetty examined the fracture surfaces of failed specimens using optical and scanning electron microscopy. These studies indicate that failures were initiated at subsurface pores (i.e., a volume defect). This type of fracture site consistently occurred in all three specimen geometries.

Once again, Eq. (5) can be used to express the functional relationship between the ranked probability of failure $\left(\mathcal{P}_{i}\right)$ and the estimate of the failure strength $\left(\hat{\sigma}_{\mathrm{i}}\right)$. Using the definition of $\mathrm{B}$ given in Eq. (17), then the following relationship exists between $\mathcal{P}_{\mathrm{i}}$ and $\hat{\sigma}_{\mathrm{i}}$

$$
\hat{\sigma}_{\mathrm{i}}=\hat{\gamma}^{*}+\hat{\beta}^{*}\left[\hat{\sigma}_{\mathrm{i}} \ln \left(1 / 1-\mathcal{P}_{\mathrm{i}}\right)\right]^{1 / \hat{\alpha}^{*}}
$$

for pure bending conditions. Here

$$
\begin{gathered}
\hat{\alpha}^{*}=\hat{\alpha}+1 \\
\hat{\beta}^{*}=\left[\left(\frac{2(\hat{\alpha}+1)}{V}\right)(\hat{\beta})^{\hat{\alpha}}\right]^{1 /(1+\hat{\alpha})}
\end{gathered}
$$

and

$$
\hat{\gamma}^{*}=\hat{\gamma}
$$

are introduced. At this point the residual defined by Eq. (10) cannot be formulated since Eq. (19) cannot be solved explicitly for the estimated ranked failure stress $\left(\hat{\sigma}_{\mathrm{i}}\right)$. However, several alternatives can be pursued to effect a solution. Margetson and Cooper (1984) indicate that the actual ranked failure stress $\left(\sigma_{\mathrm{i}}\right)$ should be substituted for $\hat{\sigma}_{\mathrm{i}}$ on the right hand side of Eq. (19). Defining

$$
\mathrm{W}_{\mathrm{i}}=\sigma_{\mathrm{i}} \ln \left(1 / 1-\mathcal{P}_{\mathrm{i}}\right)
$$

\footnotetext{
${ }^{1}$ All specimen dimensions and failure stresses in Table I (including the three-point and the pressurized-disk geometries) were obtained from a personal communication with Chao and Shetty.
} 
then Eq. (13) through (15) can be solved for $\hat{\alpha}^{*}, \hat{\beta}^{*}$, and $\hat{\gamma}^{*}$. Estimated values of the material parameters $\alpha, \beta$, and $\gamma$ would then be computed from Eq. (20) through (22). However, once the substitution of

$$
\hat{\sigma}_{\mathrm{i}}=\sigma_{\mathrm{i}}
$$

is made, Eq. (11) no longer defines the sum of the squared residuals. Exactly what is being minimized is difficult to define (an approximate residual, perhaps). However, this approximate method yields fairly good results (Duffy et al., 1991). This becomes evident in the following discussion in which results of the approximate method are compared to a more rigorous solution.

Note that Eq. (13) through (15) and Eq. (19) represent $\mathrm{N}+3$ equations in terms of $\mathrm{N}+3$ unknowns $\left(\hat{\alpha}^{*}, \hat{\beta}^{*}, \hat{\gamma}^{*}\right.$, and $\left.\hat{\sigma}_{\mathrm{i}}\right)$. The alternative solution involves finding an initial estimate of the Weibull parameters using the approach where the estimated failure strengths are substituted with the actual strength data. After computing an initial estimate of the parameters, Eq. (19) is solved numerically ( $\mathrm{N}$ times) for $\hat{\sigma}_{\mathrm{i}}$. With Eq. (12) redefined as

$$
\mathrm{W}_{\mathrm{i}}=\hat{\sigma}_{\mathrm{i}} \ln \left(1 / 1-\mathcal{P}_{\mathrm{i}}\right)
$$

then Eq. (13) and (14) are solved for updated values of $\hat{\beta}^{*}$ and $\hat{\gamma}^{*}$ (using the previous value of $\hat{\alpha}^{*}$ ). The convergence criterion given by Eq. (15) is checked. If the criterion is not satisfied, $\hat{\alpha}^{*}$ is updated, and Eq. (19) is again solved numerically for $\hat{\sigma}_{\mathrm{i}}$. This iterative process is repeated until the convergence criterion is satisfied.

Both procedures are used to estimate parameters from the fourpoint bend test data listed in Table I. The approximate method produces estimates of $\hat{\alpha}=1.55, \hat{\beta}=988.6$, and $\hat{\gamma}=559.67$. The procedure that includes the solution for $\hat{\sigma}_{\mathrm{i}}$ yields parameter estimates of $\hat{\alpha}=1.68, \hat{\beta}=861.6$, and $\hat{\gamma}=558.1$. In addition, maximum-likelihood estimators are used to obtain point estimates for a two-parameter Weibull distribution. This technique gives estimated parameter values of $\hat{\alpha}=10.2$ and $\hat{\beta}=767.8$ (with $\hat{\gamma} \equiv 0$ ). The values obtained from the two-parameter maximum-likelihood estimators differ from the values reported in Chao and Shetty (1991). They used an averaging technique proposed by Batdorf and Sines (1980) that combines data from several test specimens. The pooled data are used to compute estimates from the three- and four-point bend data. The estimated scale parameters from both configurations are averaged and, if the method of Batdorf and Sines (1980) is strictly adhered to, then the residuals from two data points are minimized. The authors feel that for this method to yield meaningful results, more than two specimen geometries are needed. The results of the maximum-likelihood estimators and both nonlinear regression methods are presented in Fig. 4, where the probability of failure is plotted as a function of the failure stress; that is, Eq. (5) is graphed using the different parameter estimates. The failure data is included using Eq. (8) to establish the vertical position of each data point. The straight line represents the two-parameter fit to the data. The nonlinear curves represent the three-parameter fit to the data. It is evident that the estimated three-parameter distributions are more efficient in predicting the failure data in the high-reliability region. Also note that there is very little difference between the two procedures used to establish the three-parameter estimates.

With the estimated Weibull parameters obtained using the procedure that includes the solution for $\hat{\sigma}_{\mathrm{i}}$, reliability predictions are made for the three-point bend and the pressurized-disk geome-

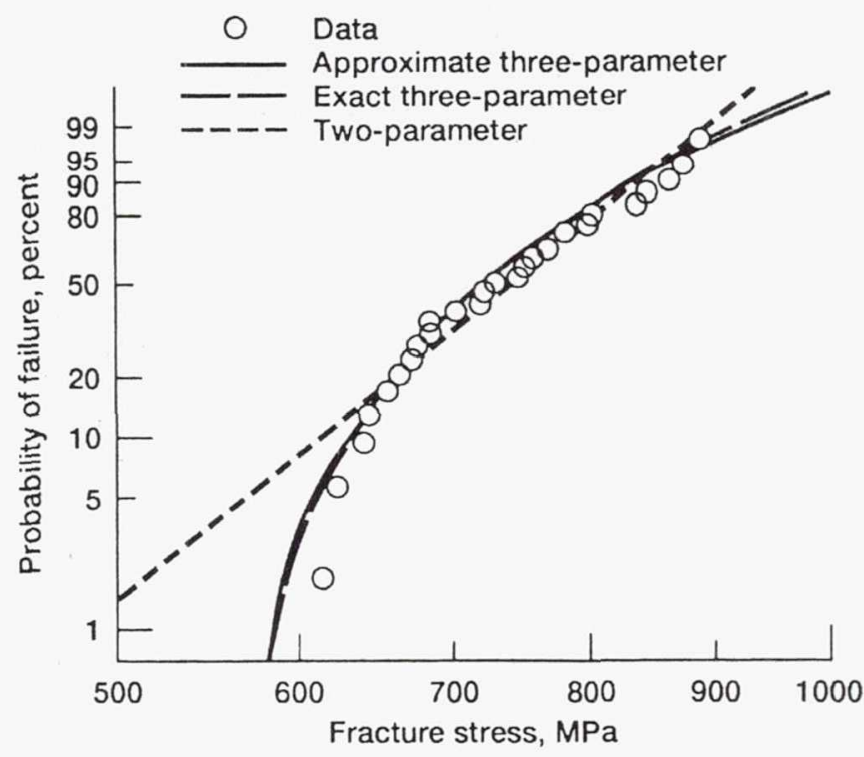

Figure 4.-Comparison of the probability of failure for the four-point specimen using the two- and three-parameter Weibull distribution.
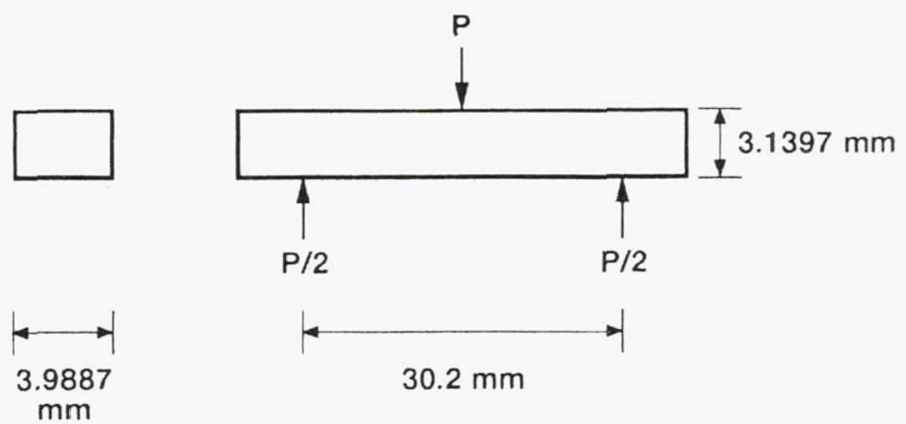

(a) Three-point bend specimen.

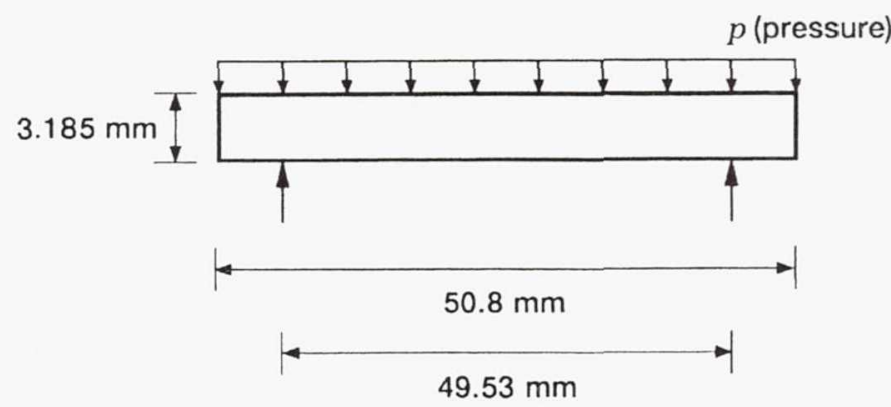

(b) Pressurized disk specimen.

Figure 5.-Geometry of the three-point bend specimen and the pressurized disk specimen.

tries used in the experimental study by Chao and Shetty (1991). Both specimen geometries are depicted in Fig. 5. The geometries are modeled using MSC/NASTRAN to determine the structural response of the specimens to mechanical loads. The three-point bend geometry is modeled with 136 eight-node elements (MSC/

NASTRAN CQUAD8). The mesh for this specimen is shown in Fig. 6. The stress distribution obtained from the finite element analysis is subsequently used as input for the integrated design 


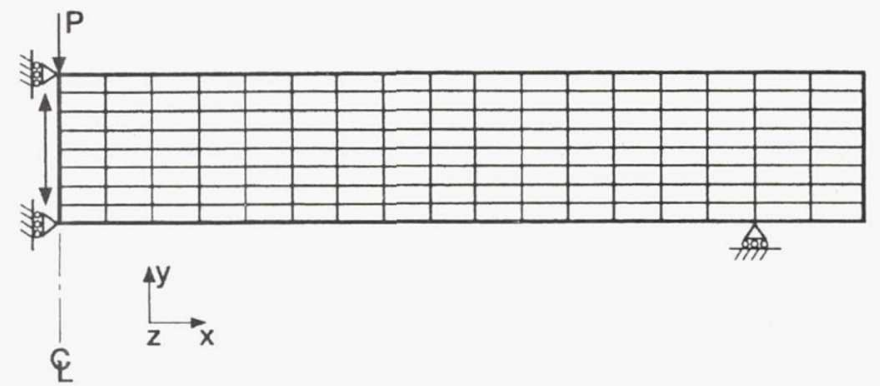

(a) Three-point bend specimen.

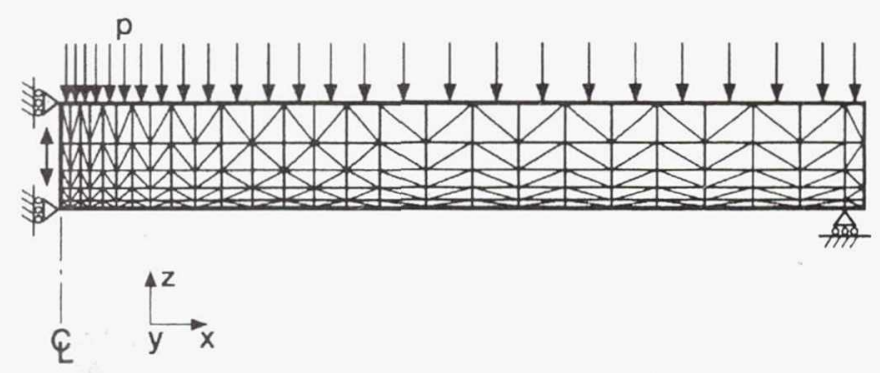

(b) Pressurized disk specimen.

Figure 6.-Finite element discretization.

program CARES (Nemeth et al., 1990). A volume flaw analysis is performed where the volume of a shell element is determined by calculating the midplane area and multiplying this value by the thickness of the element. The probability of failure curve is obtained by scaling (i.e., linearly increasing and decreasing) a single stress distribution a number of times. For each stress distribution a reliability analysis is performed with the CARES algorithm. An appropriate number of reliability computations are made to produce the nonlinear curve in Fig. 7. The linear (two-parameter) curve is established by determining the probability of failure at a single point on the curve and drawing a straight line through this point using the estimated Weibull modulus, which coincides with the slope of the linear curve. The data clearly indicates nonlinear behavior; however, both the two- and the three-parameter formulations yield conservative estimates in the high-reliability regions, but nonconservative estimates in the high probability of failure region. Both follow the trend of the data in the 5- to 60 -percent probability of failure range of the graph.

The pressurized-disk geometry is modeled with 260 six-node elements (MSC/NASTRAN CTRIAX6). The axisymmetric mesh for this specimen is also shown in Fig. 6. The probability of failure curves are depicted in Fig. 7. All probability of failure curves are generated by computing component reliability from numerous stress distributions that are obtained, once again, by linearly increasing and decreasing a single stress distribution. Here the threeparameter formulation clearly yields a better fit to the data. The two-parameter formulation is distinctly conservative at all stress levels, which can lead to overdesigned structural components. To demonstrate this, the parameter estimates obtained from the fourpoint bend data are used to compute the probability of failure of an aerospace component. Specifically, the component analyzed is a space shuttle main engine (SSME) high-pressure turbopump blade. The finite element mesh used to analyze this turbopump blade is depicted in Fig. 8. Moss and Smith (1987) used this mesh to analyze the dynamic characteristics of the blade. The mesh consists of 1025 brick elements (MSC/NASTRAN CHEXA). The shank of the blade is fully constrained. For the purpose of demonstration, it is assumed that the blade is fabricated from the monolithic silicon nitride material discussed in Chao and Shetty (1991). In the analy-

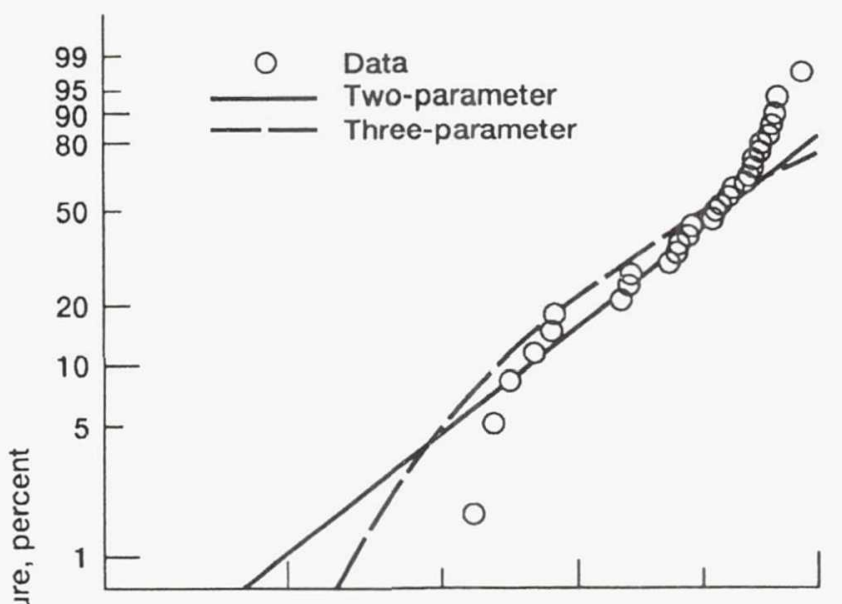

(a) Three-point bend specimen.

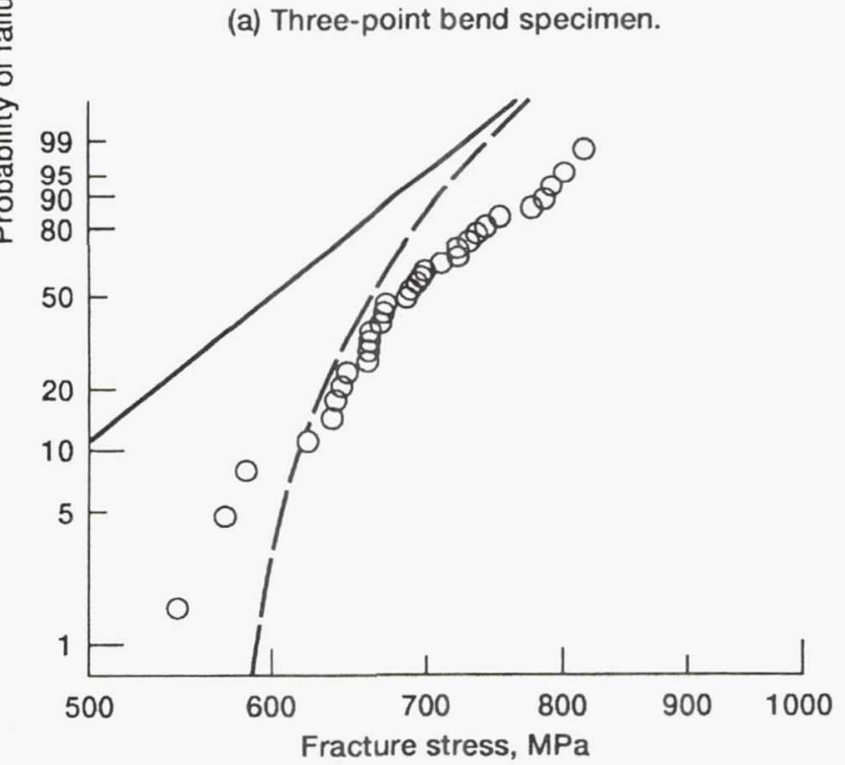

(b) Pressurized-disk specimen.

Figure 7.-CARES probability of failure results and failure data.

sis Young's modulus is taken as $285.0 \mathrm{GPa}$ and Poisson's ratio is 0.23 . The specific load case studied represents a rotational speed of $40000 \mathrm{rpm}$ at room temperature. At this rotational speed the twoparameter formulation (using the PIA model) results in a component failure probability $\left(\mathrm{P}_{\mathrm{f}}\right)$ of $\mathbf{7 5 . 2}$ percent. The three-parameter formulation results in a failure probability $\left(\mathrm{P}_{\mathrm{f}}\right)$ of .04 percent. Utilization of the monolithic material would be summarily rejected based on the limited data available and the results of the twoparameter estimates. However, the results from the three-parameter formulation could prompt further consideration. The notable difference in the probability of failure does not indicate conclusively that the underlying population is characterized by a three-parameter Weibull distribution. Additional test data may clearly demonstrate whether the underlying population is characterized by a two- or three-parameter Weibull distribution. In addition, possible design studies could result in a further reduction in the component failure probability. Whether or not further redesign would bring the component failure probability within the stringent limits established for various shuttle components is not the issue here. The authors do not advocate using monolithic silicon nitride in the fabrication of SSME turbopump blades. Rather, this aerospace example emphasizes that the common use of the two-parameter formulation can lead to extremely conservative design decisions. 


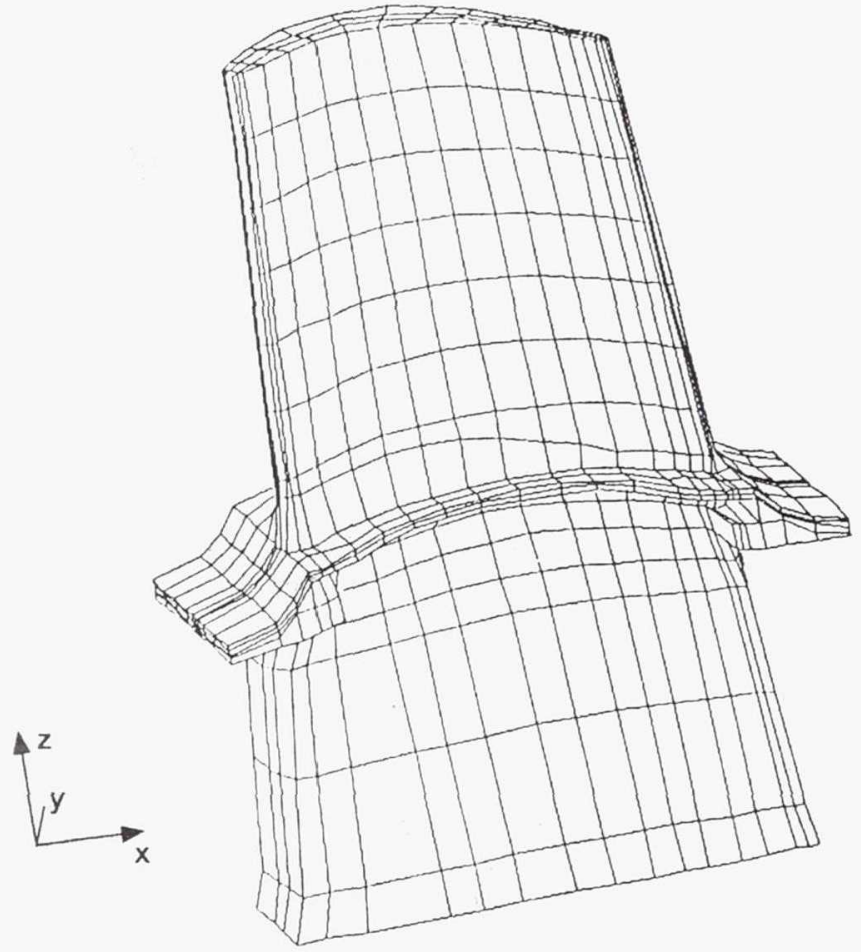

Figure 8.-Finite element discretization of turbopump blade.

\section{CONCLUSIONS}

Enough experimental data exists to suggest threshold behavior (indicated by a nonlinear behavior similar to that displayed in Fig. 4) in certain monolithics. However, whether nonlinear behavior can be attributed to the existence of a threshold stress or competing failure mechanisms is open to question because of the lack of careful fractographic analysis for most data sets (except for the Chao and Shetty data discussed previously and obtained through personal communication). This paper has reviewed a number of aspects related to the simple nonlinear regression technique proposed by Margetson and Cooper (1984). From limited numerical studies it is concluded that the estimators are well-behaved in the sense that bias is minimized, and confidence bounds tighten as the sample size is increased. However, the estimators are not invariant with respect to the underlying parameters that characterize a population. This precludes establishing exact confidence bounds and unbiasing factors.

The estimators perform reasonably well in comparison to the two-parameter maximum-likelihood estimators when both are applied to the silicon nitride data of Chao and Shetty (1991). Using an improved estimator based on the method proposed by Margetson and Cooper (1984), the three-parameter Weibull distribution easily captures the nonlinear trend of the failure data. All reliability computations are made using the simplified PIA model but better correlation to the failure data might be obtained if other more rigorous reliability models were employed. The authors are currently pursuing this analytical approach.

Although the three-parameter formulation obviously provides a better fit to the pressurized-disk data, this may not be readily evident with the three- and four-point bend data. Goodness-of-fit statistics such as the Kolmogoroff-Smirnoff statistic and the Anderson-Darling statistic should be used to establish which form of the Weibull distribution would best fit the experimental data. These approaches are currently being studied by the authors.
Finally an aerospace component is analyzed, and the results may indicate the conservativeness of the two-parameter formulation. The authors advocate the use of the three-parameter formulation of the Weibull distribution when experimental data exhibits threshold behavior. Even though the estimates proposed by Margetson and Cooper (1984) are not invariant, additional testing can be conducted to minimize the bias associated with the parameter estimates. As the reliability analysis of the SSME turbopump blade indicates, the costs from additional tests may be well worth the dramatic decrease in a component probability of failure.

\section{REFERENCES}

Batdorf, S.B., and Crose, J.G., 1974, "A Statistical Theory for the Fracture of Brittle Structures Subjected to Nonuniform Polyaxial Stresses," Journal of Applied Mechanics, vol. 41, no. 2, pp. 459-464.

Batdorf, S.B., and Heinisch, H.L., 1978, "Weakest Link Theory Reformulation for Arbitrary Fracture Criterion," Journal of the American Ceramic Society, vol. 61, no. 7-8, pp. 355-358.

Batdorf, S.B., and Sines, G., 1980, "Combining Data for Improved Weibull Parameter Estimation," Journal of the American Ceramic Society, vol. 63, no. 3-4, pp. 214-218.

Chao, L.-Y., and Shetty, D.K., 1991, "Reliability Analysis of Structural Ceramics Subjected to Biaxial Flexure," Journal of the American Society, vol. 74, no. 2, pp. 333-344.

Duffy, S.F., Palko, J.L., and Gyekenyesi, J.P., 1991, "Structural Reliability Analysis of Laminated CMC Components," ASME Paper 91-GT-210 (accepted for publication in Journal of Engineering for Gas Turbines and Power).

Margetson, J. and Cooper, N.R., 1984, "Brittle Material Design Using Three Parameter Weibull Distributions," Probabilistic Methods in the Mechanics of Solids and Structures, S. Eggwertz, and N.C. Lind, eds., Springer-Verlag, Berlin, pp. 253-262.

Midgley, E., and Pierce, F.T., 1926, "The Weakest Link Theorems on Strength of Long and of Composite Specimens," Journal of the Textile Institute, vol. 17, no. 2, pp. T355-T368.

Moss, L.A., and Smith, T.E., 1987, "Dynamic Characteristics of Single Crystal SSME Blades," Structural Integrity and Durability of Reusable Space Propulsion Systems, NASA CP-2471, pp. 211-214.

Nemeth, N.N., Manderscheid, J.M., and Gyekenyesi, J.P., 1990, "Ceramics Analysis and Reliability Evaluation of Structures (CARES), Users and Programmers Manual,” NASA TP-2916.

Quinn, G.D., 1989, "Flexure Strength of Advanced Ceramics - A Round Robin Exercise," MTL TR-89-62, (Avail. NTIS, AD-A212101).

Schneider, D., and Palazotto, A.N., 1979, “A Technique for Evaluating a Unique Set of Three Weibull Parameters Considering Composite Materials," Fibre Science and Technology, vol. 12, no. 4, pp. 269-281.

Thoman, D.R., Bain, L.J., and Antle, C.E., 1969, "Inferences on the Parameters of the Weibull Distribution," Technometrics, vol. 11 , no. 3 , pp. 445-460. 
Thomas, D.J., and Whetherhold, R.C., 1991, "Reliability Analysis of Continuous Fiber Composite Laminates," Composite Structures, vol. 18 , no. 4 , pp. 277-293.

Weibull, W.A., 1939, "A Statistical Theory of the Strength of Materials," Ingeniors Ventenskaps Akademien Handlinger, vol. 151, pp. 5-45.

Weibull, W.A., 1951, "A Statistical Distribution Function of Wide Applicability," Journal of Applied Mechanics, vol. 18, no. 3, pp. 293-297.

Weil, N.A., and Daniel, I.M., 1964, "Analysis of Fracture Probabilities in Nonuniformly Stressed Brittle Materials," Journal of the American Ceramic Society, vol. 47, no. 6 , pp. 268-274. 
Public reporting burden for this collection of information is estimated to average 1 hour per response, including the time for reviewing instructions, searching existing data sources, gathering and maintaining the data needed, and completing and reviewing the collection of information. Send comments regarding this burden estimate or any other aspect of this collection of information, including suggestions for reducing this burden, to Washington Headquarters Services, Directorate for information Operations and Reports, 1215 Jefferson Davis Highway, Suite 1204, Arlington, VA 22202-4302, and to the Office of Management and Budget, Paperwork Reduction Project (0704-0188), Washington, DC 20503.

\begin{tabular}{|l|c|r|r|}
\hline 1. AGENCY USE ONLY (Leave blank) & $\begin{array}{c}\text { 2. REPORT DATE } \\
1992\end{array}$ & $\begin{array}{r}\text { REPORT TYPE AND DATES COVERED } \\
\text { Technical Memorandum }\end{array}$ \\
\hline
\end{tabular}

\section{TITLE AND SUBTITLE}

Reliability Analysis of Structural Ceramic Components Using a

Three-Parameter Weibull Distribution

Stephen F. Duffy, Lynn M. Powers, and Alois Starlinger
5. FUNDING NUMBERS

WU $-778-32-11$
National Aeronautics and Space Administration

Lewis Research Center

Cleveland, Ohio 44135-3191
8. PERFORMING ORGANIZATION REPORT NUMBER

E-6749

9. SPONSORING/MONITORING AGENCY NAMES(S) AND ADDRESS(ES)

10. SPONSORING/MONITORING AGENCY REPORT NUMBER

National Aeronautics and Space Administration

Washington, D.C. 20546-0001

TM -105370

\section{SUPPLEMENTARY NOTES}

Prepared for the 37th International Gas Turbine and Aeroengine Congress and Exposition sponsored by the American Society of Mechanical Engineers, Cologne, Germany, June 1-4, 1992. Stephen F. Duffy and Lynn M. Powers, Cleveland State University, Cleveland, Ohio 44115 and NASA Resident Research Associate at Lewis Research Center, (work funded under NASA Cooperative Agreement NCC3-81). Alois Starlinger, National Research Council-NASA Research Associate at Lewis Research Center. Responsible person, Stephen F. Duffy, (216) 433 - 5626.

12a. DISTRIBUTION/AVAILABILITY STATEMENT

12b. DISTRIBUTION CODE

Unclassified - Unlimited

Subject Category 27

\section{ABSTRACT (Maximum 200 words)}

This paper describes nonlinear regression estimators for the three-parameter Weibull distribution. Issues relating to the bias and invariance associated with these estimators are examined numerically using Monte Carlo simulation methods. The estimators were used to extract parameters from sintered silicon nitride failure data. A reliability analysis was performed on a turbopump blade utilizing the three-parameter Weibull distribution and the estimates from the sintered silicon nitride data.

\section{SUBJECT TERMS}

Strength; Ceramic; Composites; Parameter estimates; Weibull distribution; Reliability; Confidence bounds; Bias

17. SECURITY CLASSIFICATION OF REPORT

Unclassified

\section{SECURITY CLASSIFICATION OF THIS PAGE}

Unclassified
19. SECURITY CLASSIFICATION OF ABSTRACT Unclassified
15. NUMBER OF PAGES

12

16. PRICE CODE

A03

20. LIMITATION OF ABSTRACT 